\title{
POEMS Syndrome: an Atypical Presentation with Chronic Diarrhoea and Asthenia
}

\author{
$\underline{\text { Joana Alves Vaz }}{ }^{1}$, Lilia Frada², Maria Manuela Soares ${ }^{1}$, Alberto Mello e Silva ${ }^{1}$ \\ ${ }^{1}$ Department of Internal Medicine, Egas Moniz Hospital, Lisbon, Portugal \\ ${ }^{2}$ Department of Gynecology and Obstetrics, Espirito Santo Hospital, Evora, Portugal
}

Received: 28/07/2019

Accepted: $13 / 11 / 2019$

Published: 16/12/2019

\begin{abstract}
How to cite this article: Alves Vaz J, Frada L, Soares MM, Mello e Silva A. POEMS syndrome: an atypical presentation with chronic diarrhoea and astenia.
\end{abstract} EJCRIM 2019;7: doi:10.12890/2019_001241.

Conflicts of Interests: The Authors declare that there are no competing interest

This article is licensed under a Commons Attribution Non-Commercial 4.0 License

\section{ABSTRACT}

POEMS syndrome is a rare paraneoplastic condition associated with polyneuropathy, organomegaly, monoclonal gammopathy, endocrine and skin changes. We report a case of a man with Castleman disease and monoclonal gammopathy, with a history of chronic diarrhoea and asthenia. Gastrointestinal involvement in POEMS syndrome is not frequently referred to in the literature and its physiopathology is not fully understood. Diagnostic criteria were met during hospitalization but considering the patient's overall health condition, therapeutic options were limited. Current treatment for POEMS syndrome depends on the management of the underlying plasma cell disorder. This report outlines the importance of a thorough review of systems and a physical examination to allow an attempted diagnosis and appropriate treatment.

\section{LEARNING POINTS}

- POEMS syndrome should be suspected in the presence of peripheral polyneuropathy associated with monoclonal gammopathy; diagnostic workup is challenging and delay in treatment is very common.

- Gastrointestinal involvement is not frequent and initial presentation with chronic diarrhoea is unusual and rarely mentioned in the literature.

- There is no consensus on the therapeutic approach, which can include autologous transplantation and chemotherapy. Frequently, these patients present fatigue, weight loss and sarcopenia with reduction of performance status, limiting subsequent therapeutic options.

\section{KEYWORDS}

POEMS syndrome, chronic diarrhoea, polyneuropathy

\section{CASE DESCRIPTION}

A 73-year-old man with Castleman disease and IgA lambda monoclonal gammopathy was diagnosed with unicentric involvement (cervical lymphadenopathies) and treated during the previous 6 years with corticoids and rituximab, with a complete response. At that time, no gastrointestinal or neurological symptoms were described. Surveillance was maintained, with normal annual CT tomograms. He presented to the emergency department after a 1-year history of diarrhoea (3 times/day, without blood, mucus or pus), accompanied by diffuse abdominal pain, anorexia and weight loss (10 kg - 16\% of his body weight). During the previous 2 months, he noticed symmetrical paraesthesias of the lower limbs, describing the sensation of coldness and increased peripheral oedema.

At the time of admission, on physical examination, he was apyretic, hypotensive (blood pressure $90 / 70 \mathrm{mmHg}$ ) and presented numerous angiomas in the anterior thoracic wall (Fig. 1) and digital clubbing (Fig. 2). 


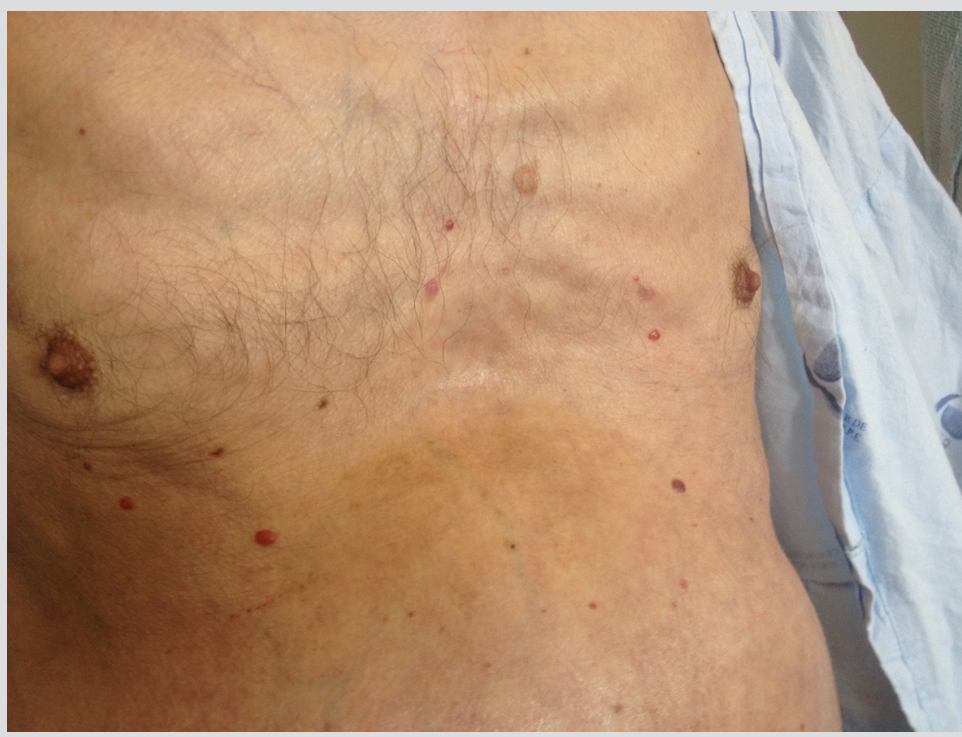

Figure 1. Angiomas in the anterior aspect of the thorax

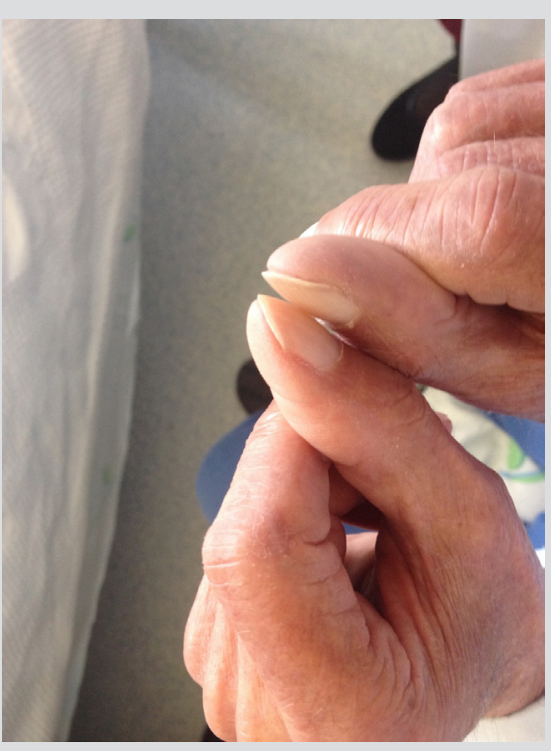

Figure 2. Digital clubbing

Cardiopulmonary examination revealed bilateral crepitations and abdominal observation was normal. Blood tests showed normocytic normochromic anaemia (haemoglobin $10.9 \mathrm{~g} / \mathrm{dl}$, mean corpuscular volume $85.9 \mathrm{fl}$, mean corpuscular haemoglobin $28.4 \mathrm{pg}$ ), thrombocytopenia $\left(122,000 / \mathrm{mm}^{3}\right)$, hypothyroidism (thyroid-stimulating hormone $9.6 \mu \mathrm{U} / \mathrm{ml}$ and free thyroxine $12 \mathrm{pmol} / \mathrm{l}$ ) and acute kidney failure (creatinine $3.9 \mathrm{mg} / \mathrm{dl}$ ). An abdominal radiogram showed diffuse distension of the colonic loops, without pneumoperitoneum or other findings. Due to renal impairment, the patient initiated fluid therapy and was transferred to the Internal Medicine department.

At the department, the patient was asthenic with marked adynamia, cachectic and dehydrated. Diarrhoea persisted ( 2 or 3 times/day) despite intensive oral and intravenous hydration, diet optimization and the introduction of probiotics. Fluid therapy improved his renal function but contributed to the development of ascites and worsening of peripheral oedema. A transthoracic echocardiogram was performed and revealed myocardiopathy with moderate mitral regurgitation, relaxation disturbance and pulmonary hypertension (estimated PASP 53.8 $\mathrm{mmHg}$.

As diarrhoea persisted for a year, the patient had already undergone some examinations, such as a colonoscopy that showed the presence of angiectasia in the ascending colon, diverticula in the sigmoid and the presence of hyperplastic polyps in the hepatic angle. After polypectomy, histologic examination revealed sigmoid tubular adenoma with low-grade dysplasia. He had a gastric endoscopy with findings of chronic gastritis of the body and erosion with decreased villi in the antrum. Testing for Clostridium difficile toxin was negative. Diagnostic workup excluded the main causes of chronic diarrhoea, such as infection, coeliac disease, inflammatory bowel disease, amyloidosis and neoplasia. Complementary studies included a negative faecal occult blood test; negative parasitological and stool culture findings for Salmonella, Shigella and Campylobacter; negative serologic tests for HIV, EBV, CMV and Toxoplasma gondii. Faecal elastase and stool fat tests were normal. Protein electrophoresis showed known monoclonal gammopathy IgA lambda (albumin 51.4\%, alpha 1 globulin 6.9\%, alpha 2 globulin $9.5 \%$, beta 1 globulin 7.0\%, beta 2 globulin 9.2\% and gamma globulin 16\%). Immunoglobulin levels were: IgA 479 mg/dl (VR 50-400), IgG 905 mg/ dl (VR 600-1500) and IgM 54.3 mg/dl (VR 50-300). Bence-Jones proteins were not detected.

Screening for coeliac disease was also negative (anti-transglutaminase antibodies IgA $2.8 \mathrm{U} / \mathrm{ml}$, anti-gliadin IgG $<0.1 \mathrm{U} / \mathrm{ml}$ ). Antinuclear antibody titres were positive (1/320), but cryoglobulins and autoantibodies were negative (anti-double-stranded DNA antibody, antineutrophil cytoplasmic autoantibody, anti-Saccharomyces cerevisiae antibody, autoantibodies against smooth muscle, anti-parietal cell antibody, anti-mitochondrial antibody, anti-liver-kidney microsomal antibody were negative).

During hospitalization, Clostridium difficile infection was excluded by serial negative stool tests. Another colonoscopy was carried out and revealed the presence of rectal sessile polyps $(8 \mathrm{~mm})$ and diverticulosis of the sigmoid colon. Biopsies showed conserved colonic cellular architecture, without granulomas or pathogens and amyloid deposit staining was negative (Fig. 3).

Abdominal CT tomograms documented homogeneous hepatomegaly (approximately $18.5 \mathrm{~cm}$ ) and homogeneous splenomegaly (spleen size of approximately $14.2 \mathrm{~cm}$ ). Plain radiographs did not show osteosclerotic lesions. Considering laboratory findings, the patient had 

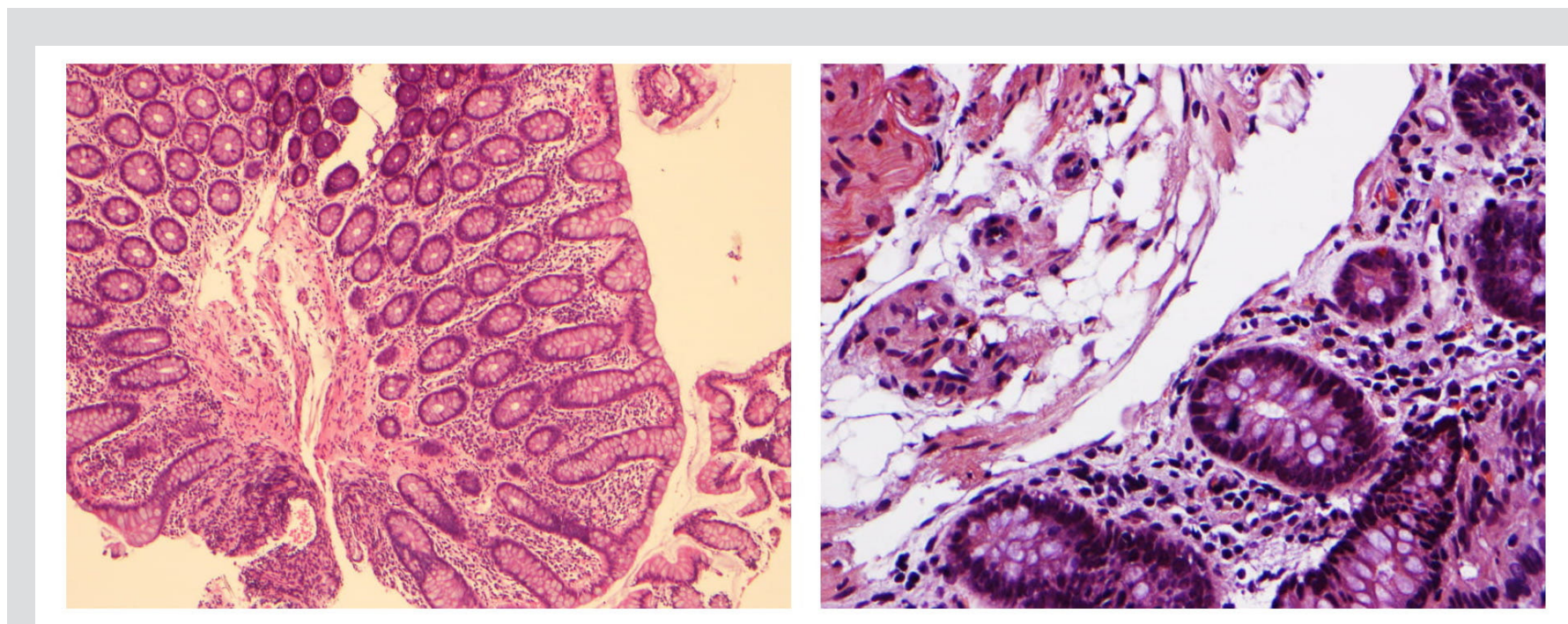

Figure 3. Colonic biopsy. Conserved colonic cellular architecture, without granulomas or pathogens. (right), x200.

hyperparathyroidism (PTHi $106.3 \mathrm{pg} / \mathrm{ml}$, corrected calcium of $8.5 \mathrm{mg} / \mathrm{dl}$ ) and vitamin D deficiency (vitamin D<20.0 nmol/l). At this point, the hypothesis of POEMS syndrome was considered to be the most likely. To confirm polyneuropathy, electromyography was carried out and documented sensory-motor demyelinating polyneuropathy with conduction blocks.

This case was appearing to fulfil all the criteria for POEMS syndrome: 2 mandatory criteria (polyneuropathy, monoclonal gammopathy), 1 major criterion (Castleman disease), and also, several minor criteria (organomegaly, endocrinopathy and cutaneous manifestations). Even considering that the most frequent endocrinopathy in POEMS syndrome is hypogonadism, serum FSH and LH levels were not tested. The patient had mild hypothyroidism, hyperparathyroidism and vitamin D deficiency.

After consulting with the patient's haematologist, it was decided to initiate corticosteroids with prednisolone $1 \mathrm{mg} / \mathrm{kg} / \mathrm{day}$, on Day 19 of hospitalization. He was started on prednisolone $60 \mathrm{mg} /$ day and was discharged 1 month later with 50 mg/day. Since corticoids were initiated, 3 days after, diarrhoea disappeared, and he maintained 1 regular daily dejection, without taking probiotics or other medications. The patient stayed under corticoids for approximately 3 months. After this, following Haematology consultation, it was decided to start him on cyclophosphamide $100 \mathrm{mg} /$ day and no more episodes of diarrhoea were present. However, due to the patient's poor performance status, no autologous transplantation, chemotherapy or immunotherapy protocol was proposed or started.

Subsequently, on follow-up visits, he gained weight, no longer suffered from diarrhoea and showed haematologic improvement (Hgb 11.7 $\mathrm{g} / \mathrm{dl}$, platelets 225,000/ul). One year after hospitalization, he had an episode of acute dyspnoea at home, died of unknown causes and an autopsy examination was not performed.

\section{DISCUSSION}

POEMS syndrome corresponds to a set of multisystemic manifestations with the acronym: polyneuropathy, organomegaly, endocrinopathy, monoclonal gammopathy and skin changes. The aetiology is related to plasmacellular dyscrasias ${ }^{[1]}$, but its pathophysiology is still not completely known. Cytokine overproduction seems to play a crucial role in this disorder, as many patients present higher levels of IL-1a, IL-6, TNF-a ${ }^{[2]}$, and more recently, VEGF. Increased levels of VEGF were related to enhanced vascular permeability, leading to associated oedema and deposition of plasma cell-derived material. VEGF is also related to disease activity but VEGF inhibition with drugs has not yielded convincing results ${ }^{[1]}$.

Diagnosis is based on clinical and laboratory findings. It is necessary to fulfil both mandatory criteria (peripheral polyneuropathy and monoclonal gammopathy) plus one of the major criteria and at least one of the minor criteria. Minor criteria of POEMS syndrome are organomegaly, extravascular volume increase, papilloedema, endocrine changes, cutaneous alterations and thrombocytosis or polycythaemia.

Our patient had hepatosplenomegaly, ascites and peripheral oedema. Dermatologic changes presented were acrocyanosis, trunk angiomas, "white nails" and digital clubbing (Fig. 2).

As a multisystemic involvement, POEMS syndrome is associated with chronic renal disease, myocardial ischaemia, increased thrombotic risk 
and pulmonary manifestations, often pulmonary hypertension ${ }^{[3]}$. Gastrointestinal involvement is probably related to cytokine production and consequent vascular damage as it is described in other organs, leading to inflammation and colitis, as described previously ${ }^{[4]}$.

There are few reported cases in the literature regarding gastrointestinal symptoms in POEMS syndrome ${ }^{[4]}$. To the best of our knowledge, this is the first case presenting these symptoms without evidence of histological colitis or amyloid mucosa infiltration. According to one case $^{[5]}$, severe diarrhoea in POEMS syndrome might be caused by secondary amyloid deposition in the gastrointestinal tract induced by chronic inflammation associated with POEMS syndrome itself or as a reaction to an underlying plasma cell disorder. In this case ${ }^{[5]}$, amyloid deposits were found in biopsies taken 1 week before the patient's death and confirmed in autopsy specimens. In our case was not possible to perform more biopsies and autopsy examination was not conducted.

Therapeutic options with respect to POEMS syndrome depend on the treatment of the underlying plasma cell disorder. There is no consensual treatment and recommendations are made based on retrospective studies and case reports by adopting some other already existing therapeutics coming from treatment of multiple myeloma, plasmacytoma or amyloidosis. Firstly, it is necessary to evaluate the existence of systemic involvement or the quantity of bone lesions. If there is no medullar infiltration and a limited number of bone lesions, patients should receive radiotherapy. Adjuvant treatment for patients with severe neuropathy can be carried out with corticosteroids or chemotherapy.

Patients with medullary involvement or no bone lesions should receive a systemic treatment. The best-response treatment based on evidence is high-dose chemotherapy followed by autologous stem cell transplantation. Autologous cell transplantation seems to be the only effective treatment for POEMS and the first choice for patients without comorbidities ${ }^{[6]}$.

When patients cannot be treated with autologous stem cell transplantation, other alternatives can present less successful outcomes: corticosteroids, alkylating agents (lenalidomide, thalidomide or cyclophosphamide). In accordance with the pathophysiology other targets have been suggested, such as anti-VEGF antibodies or other immunomodulatory targets. Although, theoretically, these agents may have an influence on disease history, results have shown an association with higher mortality, probably explained by mechanisms of massive apoptosis.

Our case illustrates the importance of a thorough review of systems and an accurate physical examination. As a starting point, the approach to our patient was haemodynamic stabilization and later directed to the aetiological study of chronic diarrhoea. Recognition and integration of several components finally led us to the correct diagnosis.

Our case presented a good clinical response to corticosteroids and did not have histologic evidence of amyloid infiltration. As there are few reported cases of this gastrointestinal involvement in POEMS syndrome, and given that the pathophysiology is not fully understood, we argue that more studies are needed for better comprehension of this condition.

\section{REFERENCES}

1. Dispenzieri A. POEMS syndrome: 2017 Update on diagnosis, risk stratification, and management. Am J Hematol 2017;92:814-829.

2. Gherardi RK, Belec L, Fromont G, Divine M, Malapert D, Gaulard P, et al. Elevated levels of interleukin-1 beta (IL-1 beta) and IL-6 in serum and increased production of IL-1 beta mRNA in lymph nodes of patients with polyneuropathy, organomegaly, endocrinopathy, M protein, and skin changes (POEMS) syndrome. Blood 1994;83:2587-2593.

3. Allam JS, Kennedy CC, Aksamit TR, Dispenzieri A. Pulmonary manifestations in patients with POEMS syndrome: a retrospective review of 137 patients. Chest 2008;133:969974.

4. Dogan S, Beyazit Y, Shorbagi A, Koklu S, Ustunel S, Guler N, et al. Gastrointestinal involvement in POEMS syndrome: a novel clinical manifestation. Postgrad Med J 2005;81:e12

5. Kihara Y, Hori H, Murakami H, Hatakeyama Y, Yoshikawa I, Hamada T, et al. A case of POEMS syndrome associated with reactive amyloidosis and Waldenstrom's macroglobulinaemia. J Intern Med 2002;252:255-258.

6. D'Souza A, Lacy M, Gertz M, Kumar S, Buadi F, Hayman S, et al. Long-term outcomes after autologous stem cell transplantation for patients with POEMS syndrome (osteosclerotic myeloma): a single-center experience. Blood 2012;120:56-62. 